\title{
ON FIXED-POINT FREE FIBREWISE MAPS OF PROJECTIVE BUNDLES
}

\author{
DAVID HANDEL
}

\begin{abstract}
Let $E$ be a vector bundle and $P(E)$ its associated projective bundle. Some necessary conditions on the characteristic classes of $E$ for existence of a fibrewise fixed-point free map $P(E) \rightarrow P(E)$ are obtained.
\end{abstract}

1. Introduction. Let $E \rightarrow B$ be an $F$-vector bundle, where $F$ denotes either the reals $\mathbf{R}$, the complex numbers $\mathbf{C}$, or the quaternions $\mathbf{H}$, and let $p: P(E) \rightarrow$ $B$ denote the associated projective bundle. We consider the question of existence of fibrewise maps $f: P(E) \rightarrow P(E)$, i.e. continuous maps such that

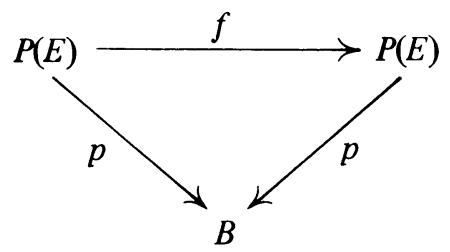

commutes, which are fixed-point free. By consideration of individual fibres, it follows, from the Lefschetz fixed point theorem, that a necessary condition for the existence of such an $f$ is that the fibre dimension of $E$ be even over $F$. The main purpose of this paper is to obtain some necessary conditions on the characteristic classes of $E$ for existence of such a map $f$. For example, we show that if the base space $B$ is simply-connected, the existence of a fibrewise fixed-point free $f: P(E) \rightarrow P(E)$ implies that all odd Stiefel-Whitney classes of $E$ vanish (Corollary 3.3). Similar results are obtained in the complex and quaternionic cases for the Chern classes and symplectic Pontrjagin classes, respectively.

Existence of a fibrewise fixed-point free map $f: P(E) \rightarrow P(E)$ is shown, in $\S 2$, to be equivalent to the existence of what we call a $P A$-structure on $E$, in analogy with the $A$-structures of James (see [1]-[3]). It is easily seen that the existence of an equivariant $A$-structure on $E$, in the sense of [1], implies the existence of a $P A$-structure on $E$, but we show in $\$ 2$ that existence of a $P A$-structure does not imply existence of an $A$-structure.

The characteristic class considerations are carried out in $\S 3$, the main tool

Received by the editors December 15, 1975.

AMS (MOS) subject classifications (1970). Primary 55F25, 55G40.

Key words and phrases. Vector bundle, associated projective bundle, fibrewise fixed-point free map, characteristic classes. 
being the structure of the cohomology ring of $P(E)$ in terms of characteristic classes of $E$.

Some examples are given in $\$ 4$. In particular, it is shown in 4.1 that the Whitney sum of two vector bundles admitting $P A$-structures need not admit a $P A$-structure. This is in contrast to the situation for $A$-structures [3, Theorem 1.4].

2. $P A$-structures. If $U$ is an $F$-vector space, we denote by $P(U)$ the projective space of $U$, i.e. the space of one-dimensional $F$-subspaces of $U$. If $u \in U-\{0\}$, let $[u] \in P(U)$ denote the one-dimensional subspace spanned by $u$. Assume that $U$ has an inner product (euclidean, hermitian, or symplectic depending on whether $F=\mathbf{R}, \mathbf{C}$, or $\mathbf{H})$. Let $S(U)$ denote the unit sphere in $U, V(U)=\left\{\left(u_{1}, u_{2}\right) \in S(U) \times S(U): u_{1} \perp u_{2}\right\}=$ Stiefel manifold of orthonormal 2-frames of $U$, and $Z(U)=\left\{\left(\left[u_{1}\right],\left[u_{2}\right]\right) \in P(U) \times\right.$ $\left.P(U): u_{1} \perp u_{2}\right\}$.

Let $E \rightarrow B$ be an $F$-vector bundle of dimension $2 n$ over $F$, with a metric (i.e. the structural group is $O(2 n), U(2 n)$, or $S p(2 n)$ depending on whether $F=\mathbf{R}, \mathbf{C}$, or $\mathbf{H})$. Form the associated bundles $p: P(E) \rightarrow B, S(E) \rightarrow B$, $V(E) \rightarrow B, Z(E) \rightarrow B$ with fibres $P\left(F^{2 n}\right), S\left(F^{2 n}\right), V\left(F^{2 n}\right), Z\left(F^{2 n}\right)$, respectively. If $x \in B$ and $E_{x}$ denotes the fibre over $x$ in $E$, then the fibres over $x$ in these associated bundles are canonically identified with $P\left(E_{x}\right), S\left(E_{x}\right), V\left(E_{x}\right)$, $Z\left(E_{x}\right)$, respectively. Moreover we have fibre bundles $q: Z(E) \rightarrow P(E)$ and $r: V(E) \rightarrow S(E)$ given by $q([u],[v])=[u]$ and $r(u, v)=u$. The fibres of these bundles are $P\left(F^{2 n-1}\right)$ and $S\left(F^{2 n-1}\right)$, respectively. In [1], an $A$-structure on $E$ is defined to be a section of $V(E) \rightarrow S(E)$.

Definition 2.1. A $P A$-structure on $E$ is a section of the bundle $q: Z(E) \rightarrow$ $P(E)$.

Proposition 2.2. There exists a fibrewise fixed-point free map $f: P(E) \rightarrow$ $P(E)$ if and only if $E$ admits a PA-structure.

Proof. If $s: P(E) \rightarrow Z(E)$ is a $P A$-structure on $E$, define $f: P(E) \rightarrow P(E)$ by $f[u]=\pi s[u]$ where $\pi: Z(E) \rightarrow P(E)$ is given by $\pi([u],[v])=[v]$. Then $f$ is fibrewise and fixed-point free.

Conversely, if $f: P(E) \rightarrow P(E)$ is a fibrewise fixed-point free map, define $s: P(E) \rightarrow Z(E)$ as follows: for $[u] \in P\left(E_{x}\right)$, let $s[u]=\left([u], \pi_{u} f[u]\right)$ where $\pi_{u}: E_{x} \rightarrow E_{x}$ is orthogonal projection on $u^{\perp}$. Then $s$ is a $P A$-structure on $E$.

Proposition 2.3. If $E \rightarrow B$ admits a PA-structure, and $f: X \rightarrow B$ is any continuous map, then $f^{*} E \rightarrow X$ admits a PA-structure.

Proof. $P\left(f^{*} E\right)=\left\{(x, y) \in X \times P(E): y \in P\left(E_{f(x)}\right)\right\}$. If $g: P(E) \rightarrow$ $P(E)$ is a fibrewise fixed-point free map, then so is $h: P\left(f^{*} E\right) \rightarrow P\left(f^{*} E\right)$ given by $h(x, y)=(x, g(y))$.

In [1], an equivariant $A$-structure on $E$ is defined to be an $A$-structure $s: S(E) \rightarrow V(E)$ such that $s(u z)=s(u) z$ for all $u \in S(E), z \in S(F)$, and it is shown there that such cannot exist unless $F=\mathbf{R}$. An equivariant $A$-struc- 
ture on $E$ yields a $P A$-structure on $E$ by passage to quotients.

Note that if $E$ is an $F$ 2-plane bundle, then the fibre of $Z(E) \rightarrow P(E)$ is a single point, and so a unique $P A$-structure exists for $E$. However, by [2, Theorem 1.2], if $E$ admits an $A$-structure, then all odd Stiefel-Whitney classes of $E$ vanish and, in particular, $E$ must be orientable. Thus real nonorientable 2-plane bundles are examples of vector bundles which admit $P A$-structures, but not $A$-structures.

3. Characteristic classes. Throughout this section $d$ will denote the dimension of $F$ over $\mathbf{R}$, and the coefficients for cohomology will be understood to be $\mathbf{Z}_{2}$ if $F=\mathbf{R}$, and $\mathbf{Z}$ if $F=\mathbf{C}$ or $\mathbf{H}$.

Let $E \rightarrow B$ be an $F$ vector bundle with a metric. Let $L(E) \rightarrow P(E)$ denote the canonical line bundle over $P(E)$, i.e. the fibre over $[u]$ consists of the points on the line $[u]$. Then $p^{*} E \cong L(E) \oplus L(E)^{\perp}$, where $p: P(E) \rightarrow B$ denotes the projection, and we can identify $Z(E)$ with $P\left(L(E)^{\perp}\right)$.

Let $\sigma_{i}(E) \in H^{d i}(B)$ denote the $i$ th characteristic class of $E$ (StiefelWhitney, Chern, or symplectic Pontrjagin depending on whether $F=\mathbf{R}, \mathbf{C}$, or $\mathbf{H})$. As is well known, the structure of $H^{*}(P(E))$ is as follows:

3.1. $H^{*}(P(E))$ is a free $H^{*}(B)$-module with basis $1, x, x^{2}, \ldots, x^{\operatorname{dim} E-1}$ where the module structure is via $p^{*}$, and $x=\sigma_{1}(L(E))$. The multiplicative structure is determined by the relation

$$
\sum_{i=0}^{\operatorname{dim} E}(-1)^{i} p^{*} \sigma_{i}(E) x^{\operatorname{dim} E-i}=0 .
$$

For an exposition see, e.g., [4, Chapter V]. We follow the sign conventions of [4].

TheOREM 3.2. Let $E \rightarrow B$ be an $F$ 2n-plane bundle with a metric. Suppose there exists a fibrewise fixed-point free map $P(E) \rightarrow P(E)$. Then there exists a class $a \in H^{d}(B)$ such that

$$
\sum_{k=0}^{2 n-1-r} \sum_{i=k}^{r+k}(-1)^{i}\left(\begin{array}{l}
i \\
k
\end{array}\right) \sigma_{2 n-1-r-k}(E) a^{k}=0
$$

for $0 \leqslant r \leqslant 2 n-2$.

Proof. By 2.2 there exists a section $s: P(E) \rightarrow Z(E)=P\left(L(E)^{\perp}\right)$ of $q: P\left(L(E)^{\perp}\right) \rightarrow P(E)$. By $3.1, H^{*}\left(P\left(L(E)^{\perp}\right)\right)$ is the free $H^{*}(P(E))$-module (via $q^{*}$ ) on $1, x, x^{2}, \ldots, x^{2 n-2}$, where $x=\sigma_{1}\left(L\left(L(E)^{\perp}\right)\right.$ ), and we have the relation

$$
\sum_{i=0}^{2 n-1}(-1)^{2 n-1-i} q^{*} \sigma_{2 n-1-i}\left(L(E)^{\perp}\right) x^{i}=0 .
$$

Since $p^{*}(E) \cong L(E) \oplus L(E)^{\perp}$, it follows from the Whitney product formula that

$$
p^{*} \sum_{i=0}^{2 n} \sigma_{i}(E)=(1+y) \sum_{i=0}^{2 n-1} \sigma_{i}\left(L(E)^{\perp}\right)
$$


where $y=\sigma_{1}(L(E))$. From (2) it follows that

$$
\sigma_{k}\left(L(E)^{\perp}\right)=\sum_{j=0}^{k}(-1)^{j} y^{j} p^{*} \sigma_{k-j}(E) \text { for } 0 \leqslant k \leqslant 2 n-1 .
$$

Substituting into (1) and factoring out $(-1)^{2 n-1}$ we obtain

$$
\sum_{i=0}^{2 n-1} \sum_{j=0}^{2 n-1-i}(-1)^{i+j} q^{*} y^{j} q^{*} p^{*} \sigma_{2 n-1-i-j}(E) x^{i}=0 .
$$

Applying $s^{*}$ to (4) we obtain

$$
\sum_{i=0}^{2 n-1} \sum_{j=0}^{2 n-1-i}(-1)^{i+j} y^{j} p^{*} \sigma_{2 n-1-i-j}(E)\left(s^{*} x\right)^{i}=0 .
$$

By 3.1 there exist unique classes $a \in H^{d}(B), b \in H^{0}(B)$ such that $s^{*} x=p^{*} a$ $+\left(p^{*} b\right) y$. Substituting into (5) we obtain

(6) $\sum_{i=0}^{2 n-1} \sum_{j=0}^{2 n-1-i} \sum_{k=0}^{i}(-1)^{i+j}\left(\begin{array}{l}i \\ k\end{array}\right) p^{*}\left[\sigma_{2 n-1-i-j}(E) a^{k} b^{i-k}\right] y^{i+j-k}=0$.

Setting $r=i+j-k$ and changing the order of summation we obtain

$$
\sum_{r=0}^{2 n-1} \sum_{k=0}^{2 n-1-r} \sum_{i=k}^{r+k}(-1)^{r+k}\left(\begin{array}{l}
i \\
k
\end{array}\right) p^{*}\left[\sigma_{2 n-1-r-k}(E) a^{k} b^{i-k}\right] y^{r}=0 .
$$

From (7) and the fact (3.1) that $1, y, y^{2}, \ldots, y^{2 n-1}$ form a free module basis of $H^{*}(P(E))$ over $H^{*}(B)$, we have

$$
\sum_{k=0}^{2 n-1} \sum_{i=k}^{r+k}(-1)^{k}\left(\begin{array}{l}
i \\
k
\end{array}\right) \sigma_{2 n-1-r-k}(E) a^{k} b^{i-k}=0
$$

for $0 \leqslant r \leqslant 2 n-1$. Taking $r=2 n-1$ in (8), we obtain $1+\sum_{i=1}^{2 n-1} b^{i}=0$, from which it follows that $b=-1$. Substituting this into (8) yields the theorem.

Corollary 3.3. If $E \rightarrow B$ is as in 3.2, and if $H^{d}(B)=0$, then $\sigma_{i}(E)=0$ for $i$ odd.

Proof. For then $a=0$, and so only the $a^{0}=1$ terms in 3.2 survive, yielding, for $0 \leqslant r \leqslant 2 n-2,0=\sum_{i=0}^{r}(-1)^{i} \sigma_{2 n-1-r}(E)$. For $r$ even, this yields $\sigma_{2 n-1-r}(E)=0$.

Corollary 3.4. Let $E \rightarrow B$ be as in 3.2. Then $\sigma_{1}(E)$ is divisible by $n$. In particular, if $F=\mathbf{R}$ and $n$ is even, $E$ must be orientable.

PRoof. Setting $r=2 n-2$ in 3.2 yields $\sigma_{1}(E)=n a$. In particular, if $F=\mathbf{R}$ and $n$ is even we obtain $w_{1}(E)=0$.

\section{Examples.}

EXAMPLE 4.1. Let $L$ denote the canonical $F$ line bundle over $P\left(F^{m}\right)$, $m \geqslant 2$, and $L_{0}$ the trivial line bundle over $P\left(F^{m}\right)$. Let $\pi_{i}: P\left(F^{m}\right) \times P\left(F^{m}\right) \rightarrow$ $P\left(F^{m}\right), i=1,2$, denote the projections on the first and second factors, respectively. Let $E_{i}=\pi_{i}^{*}\left(L \oplus L_{0}\right), i=1,2$. Since $E_{1}$ and $E_{2}$ are both 2-plane 
bundles, they both admit $P A$-structures (\$2). Write $u=\sigma_{1}(L)$. By the Whitney product formula, it follows that $\sigma_{1}\left(E_{1} \oplus E_{2}\right)=u \times 1+1 \times u$, which is not divisible by 2 in $H^{d}\left(P\left(F^{m}\right) \times P\left(F^{m}\right)\right)$. Thus by $3.4, E_{1} \oplus E_{2}$ does not admit a $P A$-structure. Thus the collection of $F$ vector bundles admitting $P A$-structures is not closed under Whitney sum.

EXAMPLE 4.2. Let $E \rightarrow B$ be any real vector bundle. Then there exists a fibrewise fixed-point free map $f: P(E \oplus E) \rightarrow P(E \oplus E)$ given by $f[u, v]=$ $[v,-u]$.

Example 4.3. Suppose $F=\mathbf{C}$ or $\mathbf{H}$, and let $L$ and $L_{0}$ be as in 4.1. Let $E=L \oplus L_{0} \oplus L_{0}$. Then $\sigma_{1}(E \oplus E)=2 u$, which is not divisible by 3 in $H^{d}\left(P\left(F^{m}\right)\right)$. Thus, by $3.4, E \oplus E$ does not admit a $P A$-structure. Thus the analogue of 4.2 for the complex and quaternionic cases is false.

\section{REFERENCES}

1. I. M. James, On sphere-bundles. I, Bull. Amer. Math. Soc. 75 (1969), 617-621. MR 39 \#3517.

2. , On sphere-bundles. II, Bull. London Math. Soc. 1 (1969), 323-328. MR 44 \#3339.

3. Bundles with special structure. I, Ann. of Math. (2) 89 (1969), 359-390. MR 39 \#4868.

4. R. E. Stong, Notes on cobordism theory, Princeton Univ. Press, Princeton, N. J.; Univ. Tokyo Press, Tokyo, 1968. MR 40 \#2108.

Department of Mathematics, Wayne State University, Detroit, Michigan 48202 